\title{
Las Pymes frente a la pandemia: El necesario desarrollo del pensamiento estratégico y de la planificación estratégica
}

doi: 10.33264/rpa.202101-06

Dr. Galvarino Casanueva Yáñez

Escuela de Administración UNIACC Facultad de Administración UNIACC

\section{Resumen}

El presente artículo constituye un estudio y análisis comparativo de los conceptos de pensamiento estratégico y planificación estratégica, basado en estudios de diferentes autores, que posibilita comprender la importancia de ambos términos y de la correcta aplicación en las empresas y que permite, fundamentalmente, a los directivos y/o dueños de las pymes y a emprendedores en general, proyectar a las organizaciones (o futuros emprendimientos) en forma sostenible en el tiempo y a evitar la mortandad en el valle de la muerte, sobre todo en la época de pandemia (Covid19) que estamos viviendo, a partir del desarrollo de innovaciones tecnológicas planeadas desde la filosofía empresarial y que involucre, como gran desafío para las pymes (pequeñas y mediana empresas), la construcción e implementación de planes de acción correctamente articulados y de la construcción de escenarios adecuados de crecimiento y desarrollo que beneficien a todos los agentes involucrados, mediante la generación de ventajas competitivas. Es decir, tiene por finalidad orientar el diseño de estrategias que permitan a las pymes ver y materializar, desde el pensamiento estratégico y de la planificación estratégica, una innovación tecnológica, que les posibilite el logro de la meta básica financiera de crecer y ser competitivas en el mercado.

Este trabajo de investigación es una parte de la tesis doctoral de su autor, doctorado en Administración Gerencial por la Universidad Benito Juárez G., que busca explicar la alta tasa de mortandad de la pequeña y mediana empresa (pyme) en la localidad de Melipilla - Chile y, asimismo, fomentar el nacimiento y desarrollo de este tipo de organizaciones mediante una: "Propuesta de valor: Un modelo de solución de negocios para las pymes chilenas".

Palabras claves: estrategia, pymes, cultura empresarial, pensamiento estratégico, planificación y ventaja competitiva.

\section{Abstract}

This article constitutes a study and comparative analysis of the concepts of strategic thinking and strategic planning, based on studies by different authors, which makes it possible to understand the importance of both terms and the correct application in companies and which, fundamentally, allows managers and / or owners of SMEs and entrepreneurs in general, project organizations (or future ventures) in a sustainable 
manner over time and avoid death in the death valley, especially in the time of pandemic (Covid19) that we are living, from the development of technological innovations planned from the philosophy business and that involves, as a great challenge for SMEs (small and medium enterprises), the construction and implementation of correctly articulated action plans and the construction of appropriate growth and development scenarios that benefit all the agents involved, through generation of competitive advantages. That is, it aims to guide the design of strategies that allow SMEs to see and materialize, from strategic thinking and strategic planning, a technological innovation, which enables them to achieve the basic financial goal of growing and being competitive in the market.

This research work is a part of the doctoral thesis of its author, PhD in Management Administration from the University Benito Juárez G., which seeks to explain the high mortality rate of small and medium enterprises (SMEs) in the town of Melipilla - Chile and also promote the birth and development of these types of organizations through a: "Value proposition: A business solution model for Chilean SMEs".

Keywords: strategy, SMEs, business culture, strategic thinking, planning and competitive advantage.

\section{Introducción}

Las pequeñas y medianas empresas (pymes) deben enfrentar complejos escenarios y diversas complicaciones durante sus primeros años de vida, tiempo que se conoce como "el valle de la muerte", período donde sólo hay dos opciones: continuar o fracasar. En Chile existe más de un millón de Pymes formales y las tres cuartas partes de éstas nacen como microempresas. Las Pymes constituyen el 52,5\% de las empresas que existen en el país y emplean al $38,7 \%$ de los ocupados. En tanto, en las microempresas estos porcentajes alcanzan al 44,4\% y $5 \%$, respectivamente. Sin embargo, el 15\% de las Pymes no alcanza a llegar al primer año de vida y queda en el camino y sólo el 40\% permanece más allá de 7 años. (Quinta Encuesta Longitudinal de Empresas/2019)

Una de las principales debilidades de las pymes chilenas es la ausencia de un pensamiento estratégico que posibilite el desarrollo de una cultura. Y la cultura es uno de los factores de éxito de cualquier empresa que actúa como elemento de cohesión, genera motivación, imprime sentido de pertenencia en sus miembros y brinda el ámbito propicio para la innovación y desarrollo del negocio. Así, el desafío de todo emprendedor y dueño de pyme es crear una cultura sólida, sustentada en su manera de ver y entender el negocio, que permita crear un ambiente entusiasta y diferenciador.

El presente trabajo es parte de una investigación realizada a las pymes de la localidad de Melipilla - Chile y que tuvo entre sus finalidades orientar el diseño de 
estrategias que permitan a las pymes ver y materializar, desde el pensamiento estratégico y de la planificación estratégica, una innovación tecnológica, que les posibilite el logro de la meta básica financiera de crecer y ser competitivas en el mercado.

La investigación ilustra la forma como las pymes pueden obtener un crecimiento y desarrollo empresarial a partir de una innovación tecnológica planeada desde su filosofía empresarial. La misma desarrolla como desafío para estas empresas, el desarrollo e implementación de un plan de acción articulado y la construcción de escenarios de desarrollo que involucren y beneficien a todos los agentes, produciéndoles ventajas competitivas y ventajas comparativas.

El estudio se realizó encuestando a 45 empresas de la ciudad de Melipilla - Chile, teniendo como táctica lo planteado por Daft (2005), quien considera que la estrategia competitiva debe ser diferente, seleccionar una serie de actividades distinta a las que otros han seleccionado, para ofrecer una mezcla única de valor. Los resultados de la investigación apuntan a que las pymes de la ciudad de Melipilla - Chile, no tienen una estructura tecnológica adecuada y no han realizado un proceso que les permita el desarrollo y comercialización de nuevos o mejorados productos con éxito. Tampoco se evidencia que estas empresas piensen las estrategias desde su filosofía organizacional poniendo en práctica el pensamiento estratégico y, desde luego, tampoco desarrollan en general una adecuada planificación estratégica.

La parte de la investigación que aquí se presenta es un análisis sobre la diferencia entre pensamiento estratégico y de planeación estratégica, aspecto que resulta fundamental para que las pymes puedan obtener un crecimiento y desarrollo empresarial a partir de una innovación tecnológica planeada desde su filosofía empresarial.

\section{Desarrollo}

El artículo 2do de la Ley 20.416 del ministerio de Economía, conocida como "Estatuto Pyme”, establece las siguientes categorías, según monto de ingresos anuales por ventas, servicios y otras actividades del giro en el último año calendario (menos el IVA e impuestos específicos): a. Las microempresas son empresas cuyos ingresos no superaron las 2.400 UF en el último año calendario; b. Las pequeñas empresas son aquellas cuyos ingresos anuales están entre $\mathbf{2 . 4 0 0}$ y $\mathbf{2 5 . 0 0 0}$ UF por ventas y servicios en el último año; c. Las medianas empresas consisten en empresas que tuvieron ingresos entre 25.000 y $\mathbf{1 0 0 . 0 0 0 ~ U F ~ e n ~ e l ~ u ́ l t i m o ~ a n ̃ o ~ c a l e n d a r i o ; ~ y , ~ d . ~ L a s ~}$ empresas grandes son aquellas que tuvieron ingresos por ventas y servicios sobre $\mathbf{1 0 0 . 0 0 0}$ UF en el último año. 
Las pymes deben enfrentar complejos escenarios y diversas complicaciones durante sus primeros años de vida, tiempo que se conoce como "el valle de la muerte", período donde sólo hay dos opciones: continuar o fracasar. Es en el "valle de la muerte" cuando muchas de las habilidades más blandas de los emprendedores deberían salir a flote. Estas son las que mantienen el grupo cohesionado, en busca de un objetivo planteado desde el día cero con una visión inamovible en sus mentes.

Es aquí donde triunfan los perseverantes, los inagotables, los que tienen una convicción tan grande de que van a triunfar y que no los detiene nada ni nadie. Son estas personas que se levantan motivadas todos los días y que, a pesar de saber que se están hundiendo cada día más, no dejan que esto merme sus esfuerzos, puesto que estaba dentro de lo planificado y no hay problema con sobrellevarlo. La determinación que debe existir en el equipo emprendedor es vital, pero también el empleo de estrategias, pensamiento estratégico y de planificación estratégica es crucial.

La conducta de los directivos y/o emprendedores (que no constituyen parte de este estudio) más el empleo de las herramientas adecuadas (que sí son el tema central del presente artículo) permiten, en forma conjunta, desarrollar un análisis estratégico que sirve para apoyar la toma de decisiones de las organizaciones respecto a lo que hacen actualmente y a lo que deberán hacer en el futuro para poder acoplarse a los cambios y a las demandas del entorno, lograr ser más eficientes (disponer de los recursos para conseguir un efecto determinado) y eficaces (lograr el efecto que se desea o se espera), y mejorar la calidad en los bienes/servicios que ofrecen.

Ahora, frente a la pandemia, múltiples estudios recientes muestran cómo las PYMEs han sufrido profundamente con la crisis del COVID 19. Gourinchas et al (2020) estiman, para una muestra de 17 países, que la tasa de quiebras de PYMEs ha aumentado en 9 puntos porcentuales, en ausencia de apoyo gubernamental, lo que podría atenuarse en 0.7 puntos de PIB y $3 \%$ de empleo si se apoya a las industrias perjudicadas con ayudas directas. En efecto, Humphries et al (2020) constatan que las expectativas de crecimiento para las pequeñas empresas 14 americanas se han deteriorado por dificultades en el acceso a ayudas gubernamentales. Fairlie (2020) presenta evidencia para PYMEs de Estados Unidos, de que al primer mes de restricciones sanitarias la cantidad de empresas activas de menor tamaño cayó un $22 \%$, recuperándose ligeramente en el mes siguiente. Por lo tanto, la evidencia indica que las PYMEs no solo han sido particularmente afectadas por la pandemia, sino que, además, el acceso a ayudas no ha sido necesariamente el óptimo.

\section{Estrategia}

Una estrategia se define como los principales cursos de acción que se seleccionan e implementan para obtener uno o más objetivos y, desde luego, uno de los desafíos 
más relevantes al crear estrategias es que sean únicas en relación con los competidores. La planeación estratégica constituye el marco referencial de tipo general proyectado para la obtención de los objetivos institucionales de la organización, y tiene como objetivo básico el establecimiento de guías generales de acción de ésta. Siguiendo a Hill y Jones (2009) una estrategia es un conjunto de acciones estructuradas que los administradores adoptan para mejorar el desempeño de su compañía.

Por otro lado, David (2007) define la administración estratégica como el arte y la ciencia de formular, implementar y evaluar decisiones multifuncionales que le permitan a una organización lograr sus objetivos. Hax y Majluf (1995) señalan que la estrategia pasa a ser un marco conceptual fundamental a través del cual una organización puede afirmar su continuidad vital, a la vez que facilitar su adaptación a un medio cambiante.

Dentro de las características principales encontradas en el estudio del concepto de estrategia tenemos:

$>$ Que se genera a largo plazo

$>$ Su flexibilidad

$>$ Que se basa en el principio del compromiso

Abarca a la empresa en su totalidad

Por otro lado, también se determinan los elementos que la forman:

Definir la misión y visión de la empresa

$>$ Fijar los objetivos y metas de la empresa

$>$ Crear estrategias y procedimientos

$>$ La calendarización y presupuesto

$>$ Construcción y establecimientos de políticas y reglamentos. 


\section{Tabla 1}

Comparación entre planificación

tradicional y planificación

estratégica

\begin{tabular}{|c|c|c|}
\hline $\begin{array}{l}\text { ELEMENTO DE } \\
\text { COMPARACIÓN }\end{array}$ & PLANIFICACIÓN TRADICIONAL & $\begin{array}{l}\text { PLANIFICACIÓN } \\
\text { ESTRATÉGICA }\end{array}$ \\
\hline Origen & Antes de 1970 & A partir de 1975 \\
\hline Valor privilegiado & Eficiencia & Eficacia \\
\hline Sistema & Cerrado & Abierto \\
\hline Proceso & Inductivo & Deductivo \\
\hline Medio ambiente & Estable & Cambiante \\
\hline Información & Cuantitativa & $\begin{array}{l}\text { Cuantitativa } \\
\text { Cualitativa }\end{array}$ \\
\hline Ciclo & Largo y discontinuo & $\begin{array}{l}\text { Mediano, corto } \\
\text { continuo }\end{array}$ \\
\hline Finalidad & Plan estático & Plan evolutivo \\
\hline Producto & $\begin{array}{l}\text { Decisiones orientadas hacia } \\
\text { el futuro }\end{array}$ & $\begin{array}{l}\text { Decisiones actuales } \\
\text { partir del futuro }\end{array}$ \\
\hline Ambiente & Interno & Interno - Externo \\
\hline
\end{tabular}

Fuente: Elaboración propia.

\section{Pensamiento Estratégico}

Podemos definir pensamiento estratégico como la base para los procesos de toma de decisiones estratégicas, ya que busca garantizar la viabilidad (o supervivencia) a largo plazo de la empresa. Un pensador estratégico eficiente y eficaz, tiene la capacidad de unir sus acciones cotidianas con los objetivos a largo plazo de la empresa. Davis (2007) nos plantea que el proceso de la administración estratégica puede describirse como un enfoque objetivo, lógico y sistemático para tomar decisiones importantes en una organización. El mismo autor más adelante manifiesta que: La mayoría de las personas reconocen, con base en experiencias pasadas, su buen juicio y 
sus sentimientos, que la intuición es esencial para tomar buenas decisiones estratégicas. En relación con la idea de integrar la intuición y el análisis, David (2007) señala en la toma de decisiones, escoger entre un enfoque intuitivo $u$ otro analítico no es una elección excluyente. Por otro lado, Morrisey (1996) nos plantea la interrogante ¿Quiénes son sus pensadores estratégicos y cómo deben funcionar? diferenciando el acto del pensamiento estratégico del proceso de la administración estratégica.

Dentro de las características principales del pensamiento estratégico nos encontramos con:

$>$ Proporciona las bases para la planeación estratégica

$>$ Lleva a la perspectiva

$>$ Los directivos y/o gerentes con mayores éxitos serán aquellos capaces de desarrollar un pensamiento estratégico innovador al interior de su organización

El análisis anterior nos permite encontrar y destacar las diferencias entre planeación y pensamiento estratégicos.

Así, la planeación estratégica:

$>$ Identifica y entrega Factores Críticos de Éxito (FCE)

$>$ Muestra donde se encuentra la empresa y a dónde quiere llegar

$>$ Desarrolla análisis FODA

De acuerdo con Armijo (2009) la Planificación Estratégica, PE, es una herramienta de gestión que permite apoyar la toma de decisiones de las organizaciones en torno al quehacer actual y al camino que deben recorrer en el futuro para adecuarse a los cambios y a las demandas que les impone el entorno y lograr la mayor eficiencia, eficacia, calidad en los bienes y servicios que se proveen.

Por otro lado, el pensamiento estratégico:

$>$ Define los Factores Críticos de Éxito (FCE) adecuados para los diferentes escenarios previstos

- Evalúa diferentes escenarios con el fin de obtener el fin empresarial

$>$ Su fórmula: $1 \mathrm{FCE}+1 \mathrm{FODA}=3$ veces más probabilidad del logro de objetivos

El gran aporte de los estudiosos sobre la temática bajo análisis consiste en que se clarifica un término responsable de producir una estrategia organizacional y, por lo tanto, de gran importancia en el pensamiento estratégico: La visión.

El pensamiento estratégico no constituye un conjunto de teorías, sino un punto de vista: Es el punto de vista del estratega de los negocios que ve el mundo de modo diferente.

El estratega visualiza las actividades de las empresas tal como se desarrollan en el mercado competitivo, no como las comprende la gerencia. El estratega ve la 
empresa desde el exterior, desde afuera, como si sus tareas internas se proyectaran en una gran pantalla por medio de una linterna mágica ubicada en su centro. Ello, a diferencia con el gerente operativo, quien ve a la empresa de adentro hacia afuera. Cambiar el punto de vista propio es esencial para el desarrollo del pensamiento estratégico. El punto de partida más lógico quizá sea la definición de la estrategia en sí (Gerstein, 1988).

La planeación estratégica define dónde hoy se encuentra la empresa y dónde en un "futuro", dónde se anhela que la organización se encuentre y cuándo. Indudablemente, esto conlleva a establecer el espacio temporal dentro del cual se moverá la organización. Es necesario el paso anterior para no caer en un concepto abstracto de la planeación estratégica y, por ende, el análisis del FODA también será abstracto.

\section{MODELO DE PENSAMIENTO ESTRATÉGICO}

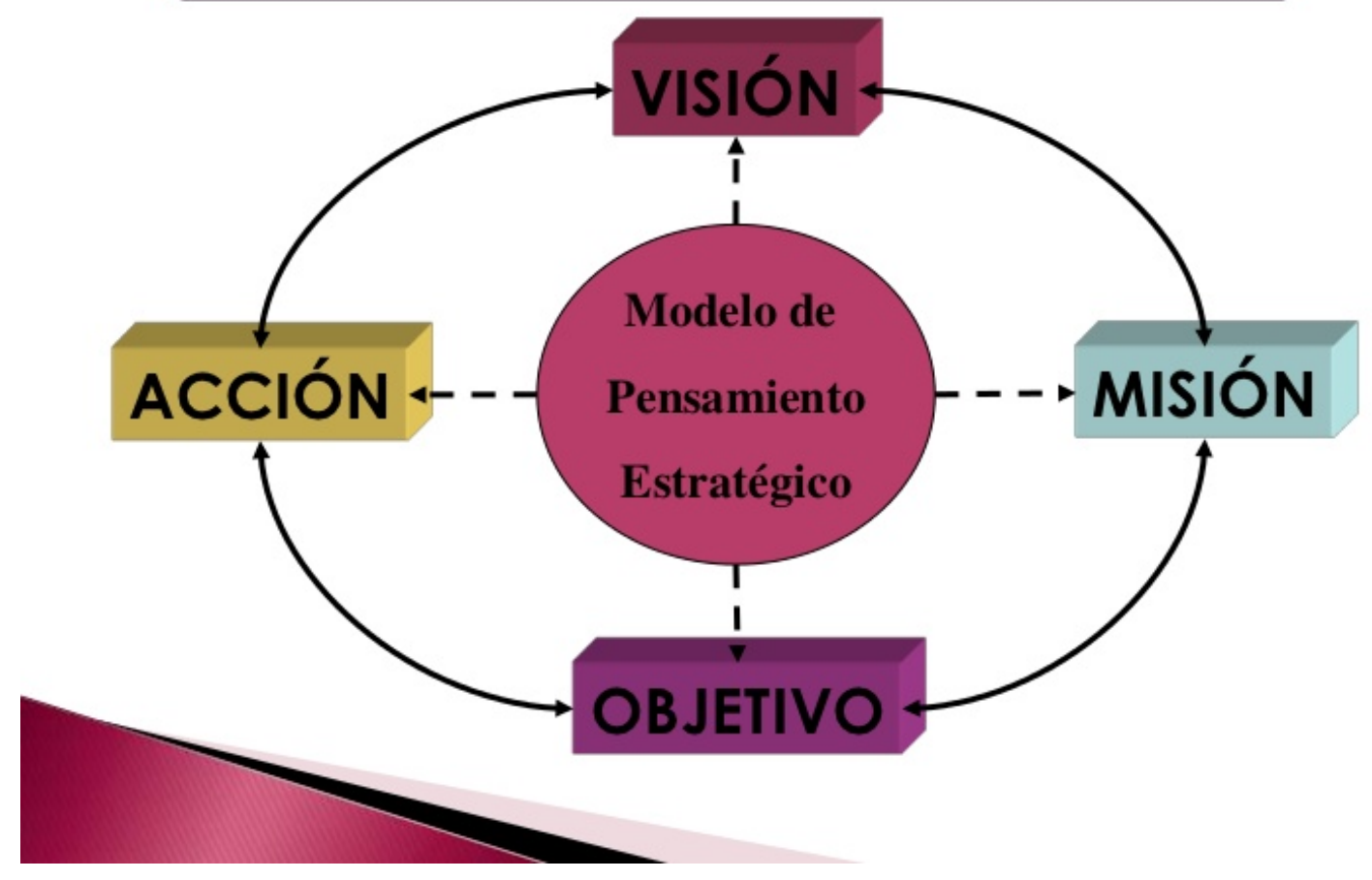

¿Por qué la importancia? Es muy necesario para que los directivos y profesionales de la empresa mediante sus ideas, pensamientos y acciones coloquen en movimiento al pensamiento estratégico. Justamente, son estos colaboradores quienes van a cuestionarse el cómo; es decir, como van a lograr mejorar el nuevo posicionamiento al que aspiran para su empresa en el tiempo establecido. Estructurado esto, ellos evaluarán los diferentes escenarios dentro de los cuales podrán avanzar como organización para lograr los fines deseados. De acuerdo con González (2011) el 
pensamiento estratégico es útil para elaborar planes o programas diseñados para alcanzar metas futuras y usar los recursos disponibles. El desarrollo de una estrategia permite diseñar enfoques, que ayudan a hacer frente a los retos planteados por el futuro.

El escenario que definen los estrategas debe contener Factores Críticos de Éxito (FCE) para que sean utilizados en la planeación estratégica, y que son diferenciadores propios de ese contexto. Indudablemente, estos FCE son fundamentales y determinantes para alcanzar los resultados, y si la empresa no los tiene o solamente los tiene en forma parcial, eventualmente solo podrá alcanzar parte de los resultados, pero muy difícilmente su totalidad. Caso contrario, el hecho de determinar cuáles son cada uno de esos factores permitirá la viabilidad, a partir de ese momento, de desarrollar un análisis FODA para cada uno de ellos. Se comprende que las empresas que desarrollan y aplican planeación estratégica basada en esta fórmula encuentran que es explosiva.

Congruente con lo anterior, la fórmula del Pensamiento estratégico es: 1 FCE + 1 FODA de ese factor $=3$ veces más probabilidad de logro

La fórmula muestra la importancia de desarrollar un FODA en abstracto que hacerlo direccionado para potenciar un factor determinante de los resultados (FCE). ¿Es factible identificarlos matemáticamente? Sí, y, de hecho, se ha creado una plantilla en Excel que posibilita y facilita los cálculos y permite una rápida identificación de éstos (Club Tablero de Comando).

Siguiendo a González (2011) los planes estratégicos son procesos de reflexión por parte del conjunto de agentes interesados en un proceso concreto (la ciudad, un sector específico de ella, un territorio, etc....) mediante el cual definen el futuro que desean desarrollar, las bases sobre las que sustentarlo, y proyectos concretos a ejecutar a lo largo del horizonte temporal definido.

El pensamiento estratégico se puede emplear en cualquiera organización que tenga por objetivo obtener y mantener una ventaja competitiva. Está fundamentado en un enfoque en la mejora continua, y es básicamente mediante la innovación y creatividad donde el pensamiento estratégico elabora una visión de futuro de una empresa antes de desarrollar un plan estratégico.

Es a través del pensamiento estratégico que los directivos pueden capturar las oportunidades actuales que ofrece el mercado y llevar a la empresa hacia un nuevo y más exitoso futuro. Esto también permite el desarrollo e implementación de un modelo de negocios en la cual la estrategia se divide en dos áreas, por un lado, se pueda pensar dentro de un proceso innovador, creativo e inteligente que estimula un 
intercambio abierto de ideas y soluciones para enfrentar desafíos muy dinámicos y a menudo impredecibles que se confrontan en la economía actual (Strategic thinking) $y$, por otro lado, como una aplicación sistemática y lógica de las estrategias que aborda una amplia gama de enfoques de gestión, incluyendo el desarrollo e implementación de un plan estratégico de la empresa (Strategic planning).

El pensamiento estratégico es una forma diferente de pensar que utiliza la intuición y la creatividad y, logra como resultado, una óptica integrada de la empresa. Se construye y desarrolla como una actividad de pensamiento individual que favorece a la empresa y su propósito, indudablemente, es identificar estrategias competitivas para posicionar significativamente y de una manera diferente en relación con la situación actual de la empresa. Según González (2011) las tres características del pensamiento estratégico que vale la pena sean consideradas por separado son: cambio, innovación e improvisación.

\section{Conclusiones}

El presente estudio nos permite encontrar una de las principales causas de la alta mortandad de las Pymes chilenas, y muy en especial en esta época de pandemia (Covid19): la no comprensión, diferenciación y aplicación del pensamiento estratégico y de la planificación estratégica por parte de estas organizaciones económicas. El termino estrategia aplicado a las empresas se orienta a seleccionar y tomar decisiones, a poder competir y a ser sostenibles en el tiempo. La capacidad del pensamiento estratégico se asocia a pensar con estrategia, a reflexionar en forma gradual y muy ordenada para evitar y/o prevenir, en lo posible, las malas decisiones para que la empresa sea viable económica y financieramente a través del tiempo. Esta cualidad de reflexión faculta tener los objetivos claros, determina la forma de alcanzarlos y establece las necesidades de recursos que ello involucra.

Así, la planeación estratégica:

$>$ Proporciona Factores Críticos de Éxito (FCE)

$>$ Señala donde se encuentra la organización y a dónde quiere llegar

> Realiza análisis FODA (Fortalezas, Oportunidades, Debilidades y Amenazas)

$\mathrm{Y}$, el pensamiento estratégico:

$>$ Define los FCE (Factores Críticos de Éxito) adecuados para los escenarios previstos

$>$ Evalúa diferentes escenarios con el afán de lograr el fin organizacional

$>1 \mathrm{FCE}+1 \mathrm{FODA}=3$ veces más probabilidad de logro de objetivos

Pensar estratégicamente no significa lo mismo que la elaboración de un plan estratégico. La diferencia entre ambos términos es evidente: El pensamiento estratégico debe considerarse como fundamental para crear, desarrollar y mantener Revista Pensamiento Académico de la Universidad UNIACC 
una ventaja competitiva, y es la herramienta de liderazgo que la gran mayoría de las empresas de la competencia buscan tener, donde se puede desplazar una empresa para obtener mejoras, que permita innovar, optimizar, y lograr una mayor productividad.

El pensamiento estratégico se desarrolla e implementa habitualmente en zonas de resolución de problemas y de toma de decisiones y con la idea de reflexionar e imaginar el devenir de la empresa. En cambio, el plan estratégico se relaciona con el análisis y poder estabilizar y formalizar sistemas y procedimientos. En sí constituye una guía deliberada y formal de las metas y objetivos de la empresa, como el medio fundamental por el cual una organización desarrolla una ventaja competitiva. El formato metodológico de planificación tradicional nos señala que el desafío de establecer la dirección estratégica es básicamente analítico: Análisis de fortalezas, debilidades, oportunidades y amenazas. Constituye una actividad fundamental a nivel del entorno como dentro de la organización. $\mathrm{Y}$, como resultado, permite seleccionar e implementar la estrategia.

Así, mientras que la planificación es una función incorporada de la toma de decisiones en general de la organización, el pensamiento estratégico necesita una síntesis mayor de tareas creativas e innovadoras al construir una visión que son diferentes a la que se tiene en la actualidad. La planificación estratégica se fundamenta en el pensamiento racional, lineal y es empleado para permitir el desarrollo solo dentro de una posición estratégica aceptada.

El pensamiento estratégico es un proceso eminentemente reflexivo que permite establecer la intención y el perfil estratégico de lo que una empresa buscar "llegar a ser". Tiene por meta encontrar y desarrollar la capacidad de anticipación de los sucesos, visualizar un destino y desarrollarlo, y alcanzar el "mañana" que se piensa es más favorable para la empresa. Constituye un pensamiento flexible, que permite, inclusive, reorganizar todos los recursos cuando el objetivo se pierde de vista, y también es capaz de readecuarse frente a los cambios de escenarios.

Por otro lado, desarrollar e implementar una estrategia, un plan más o menos detallado, en concordancia a los objetivos planteados y a una filosofía, permite anticiparse a los obstáculos y solucionarlos antes de que se presenten.

Mi pensamiento al respecto, y concuerdo con la mayoría de los autores en esta temática, es que la idea básica es desarrollar un pensamiento estratégico y después planear cómo desarrollar ese pensamiento estratégico. Empleando estas herramientas en formar eficiente y eficaz les permitirán a las pymes, con una mayor probabilidad de éxito, evitar caer en "el valle de la muerte", que es el término que se ha acuñado en las escuelas de negocios para describir el momento en que los 
emprendedores se quedan sin recursos de financiación para continuar con su negocio y que más que un lugar, es un momento en el tiempo del proceso emprendedor en el que, si no se actúa apropiadamente, las empresas quiebran y cierran. Las pymes que evitan caer en este estado son capaces de utilizar herramientas para el análisis estratégico, de manera que sean capaces de identificar problemas/necesidades a la hora de desarrollar e implementar un plan estratégico, y que además sean capaces de definir los objetivos prioritarios para solucionar dichos problemas/necesidades, que permitan, a su vez, definir la estrategia y las acciones que se llevarán a cabo para lograr dichos objetivos, ya sea a mediano o a largo plazo, pero antes requieren desarrollar necesariamente el pensamiento estratégico.

\section{Referencias}

Armijo, M. (2009). Manual de Planificación Estratégica e Indicadores de Desempeño en el Sector Público. ILPES/CEPAL

Certo, S. (2001). Administración moderna ( $8^{\circ}$ ed.). Prentice Hall.

David, F. (2007). Conceptos de Administración Estratégica (11 ed.). Pearson-Prentice Hall.

Gerstein, M. (1988). Pensamiento Estratégico: Encuentro con la tecnología, estrategias y cambios en la era de la información. Addison-Wesley.

González, M. (2011). El pensamiento estratégico como motor de la gestión de cambio de territorio.

Gourinchas, P.-O., Kalemli-Özcan, Ș., Penciakova, V., y Sander, N. (2020). Covid-19 and SME Failures. Tech. rep., National Bureau of Economic Research.

Hax, A. y Majluf, N. (1995). Gestión de Empresa con una visión estratégica. Ediciones Dolmes.

Hill, C. y Jones, G. (2009). Administración Estratégica. ( $8^{\circ}$ ed.). McGraw Hill.

Humphries, J. E., Neilson, C., y Ulyssea, G. (2020). The evolving impacts of COVID-19 on small businesses since the CARES Act.

Morrisey, G. (1993). El pensamiento estratégico. Construya los cimientos de su planeación. Prentice Hall.

Pensamiento Estratégico o Planeamiento estratégico. (2015,octubre). Club Ensayos. https://www.clubensayos.com/Temas-Variados/Pensamiento-Estrategico\%C3\%B3-Planeamiento-Estrategico/2849640.html 
Prof. Mario Héctor Voguel (2013, 2 de julio). Diferencia entre Planeacion Estrategica y Pensamiento Estrategico. YouTube. https://youtu.be/sKsuMMbtgKU

\section{Galvarino Casanueva Yáñez}

PhD en Administración Gerencial por la Universidad Benito Juárez G., México PhDœ en Administración de Empresa, Université Libre Des Science De L' Entreprise Et Des Technologies de Bruxelles, Máster en Ciencias de la Administración Université Libre Des De L’ Entreprise Et Des Technologies de Bruxelles, Master Financial Professional (AAFM), Magíster en Finanzas Corporativas por la Universidad Viña del Mar, Ingeniero Comercial (Universidad Católica del Norte, Licenciado en Ciencias de la Administración (Universidad Católica del Norte), Diplomado en Marketing Estratégico (Universidad Benito Juárez G), Diplomado en Planificación y Gestión Financiera (Universidad Benito Juárez G), Diplomado en Finanzas Corporativas (Universidad Benito Juárez G), Diplomado en Docencia Universitaria (Universidad del Pacífico). Docente en UNIACC, IACC e IPChile. Relatorías y asesorías a Pymes. Conferencista internacional de la UBJ. Docente en el Doctorado de Dirección de Proyectos de la UBJ de México.

https://orcid.org/0000-0002-6154-1551

Email:yalvary@gmail.com 